\title{
Rotation and Magnetic Fields of Solar-Like Stars
}

\author{
J.-F. Donati \\ Observatoire Midi-Pyrénées, 14 Av. Belin, 31400, Toulouse, France
}

\begin{abstract}
In this paper, I review most of the current knowledge about the strong correlation observed between the rotation rates and the magnetic fields of cool active stars. I concentrate in particular on the most recent observational results, derived from studies on dynamo field generation in stellar convective envelopes from magnetic imaging of very active rapidly rotating low mass stars. I also mention the latest attempts at investigating the large scale magnetospheric structure of these objects from extrapolations of the measured photospheric field maps, as well its dependence/influence on rotation. I finally discuss the new discovery of the feedback effects that dynamo magnetic fields generate on the global dynamics of stellar convective envelopes.
\end{abstract}

\section{Introduction}

Despite considerable progress obtained over the last century, astrophysicists are still far away from a thorough understanding on how magnetic fields are produced in the Sun. One can probably ascertain rather securely that dynamo action in the Sun requires both non axisymmetric turbulence and differential rotation, and results from the interplay of rotation and cyclonic convection in a thin interface layer at the base of the convective zone. While differential rotation produces a toroidal field structure from an initially poloidal field, turbulence then reacts on this toroidal field to turn it into a poloidal field of opposite polarity; another two such steps brings back the initial field structure and completes the cycle usually referred to as the solar magnetic cycle. Ample evidence also exists that most of these phenomena occur within a thin layer at the base of the convective zone, as the only place where rotation gradients are strong enough to produce efficiently the toroidal field and where this field can be stored for a long enough timescale compared to the the duration of the activity cycle.

It nevertheless remains clear that a lot of crucial issues concerning our understanding of the solar dynamos still remain basically unsolved. For instance, the detailed physical mechanism that leads to the regeneration of the poloidal field is still essentially a matter of speculation (e.g. Dikpati \& Gilman 2001 and references therein). It is not completely clear either which processes contribute most to the production of magnetic flux; while the smallest magnetic features in the photosphere seem to be those that carry most of the magnetic energy out of the Sun, it is not at all obvious whether this small scale field is also produced by the same dynamo processes, or some other one, taking place at a different location in the convective zone.

More generally, one is interested in how magnetic fields interact with the rotation of a star like the Sun, not only through the physical processes that 
amplify these fields and make them time variable (e.g. convection, turbulence, circulation) but also through all other processes taking place within the stellar interior and circumstellar environment (mass loss, accretion, diffusion) known to play a significant role in stellar evolution, and in particular in the temporal evolution of stellar rotation. Observing cool active stars other than the Sun is therefore very useful to provide hints and checks for any new general dynamo theory that could predict not only the magnetic stellar topologies but also the internal distribution of angular momentum and the whole rotation history of low-mass stars. In particular, the recent possibility of reconstructing brightness and magnetic images of the surfaces of rapidly rotating cool stars now offers the possibility of testing such theories in much more details than what was previously possible with older observations, usually limited to no more than checking the dependence of activity indexes with rotation rate.

In this paper, I first recall the basics of our knowledge on how magnetic activity correlates with stellar rotation rate (Sect. 2). I also summarise the new insights on this topic provided by observations of magnetic topologies of low mass stars (Sect. 3), discuss the latest results on the potential feedback of magnetic fields on the internal rotation of active stars (Sect. 4) and conclude on the most promising research avenues in this field (Sect. 5).

\section{Magnetic activity versus rotation}

By analogy with the Sun, magnetic activity in stars is usually defined as the presence of excess emission in a number of spectral lines known to form in the high temperature layers of outer stellar atmospheres (chromosphere, transition region and corona), whose existence is commonly attributed to a large scale magnetic field produced through dynamo processes. This activity, quantified by the amount of flux (relative to the total stellar luminosity) radiated in these spectral lines, is found to be ubiquitous in cool stars with convective envelopes immediately beneath the surface and has long been known to be intimately connected with stellar rotation. Most cool stars define indeed clear relationships between activity and rotation rate (e.g. Vaughan \& Preston 1980), almost irrespectively of their spectral type and duplicity (e.g. Strassmeier et al. 1990). An even better correlation (and in particular a weaker dependence with the luminosity class) is found between the level of activity and the inverse Rossby number of low-mass stars (Noyes et al. 1984). This dependence is usually interpreted as evidence that chromospheres of cool stars progressively cover a higher fraction of the stellar surface as the rotation rate increases.

More recent studies also made it clear that the activity level seems to saturate in stars with inverse Rossby numbers larger than about 1 to 10 (depending on the spectral lines used); this is usually interpreted as the evidence that, for the most rapid rotators in this stellar class, the high temperature atmosphere has already filled the largest fraction of the available circumstellar volume and can no longer grow bigger as the rotation rate (and thus the magnetic field) increases. The X-ray flux measured for these stars, corresponding to emission from high excitation coronal lines in this wavelength domain, is also observed to scale fairly well with the inverse Rossby number (Mangeney \& Praderie 1984), up again to about the same level above which it saturates. Another, yet un- 
clear, phenomenon (usually referred to as supersaturation) is the observation that this emission flux decreases again above inverse Rossby numbers of about 100 (Prosser et al. 1996).

This strong dependence between activity and rotation is interpreted by dynamo theorists by the fact that global magnetic fields generated by dynamo processes are stronger for faster rotators, and thus that surface magnetic fields cover a larger fraction of the stellar photosphere. The first estimates of magnetic fields on cool stars obtained through non polarimetric data, although unable to provide information on the field topology, nevertheless succeeded in indicating that magnetic flux from these stars roughly scaled up with their rotation rates (e.g. Saar 1996). More specifically, the result obtained from such experiments is that the surface magnetic field strength in these stars is fixed to the equipartition value while the relative fraction of the stellar surface covered with this field increases roughly linearly with the inverse Rossby number up to a saturation limit at which most of the surface is covered with fields; above this threshold, the magnetic strength starts to increase, ensuring a continuous growth in the magnetic flux with stellar rotation rates. This observational picture suggests that, as expected by dynamo theories, magnetic activity is essentially controlled by magnetic flux, which in turn depends mostly on stellar rotation.

Although promising, this simple picture is however poorly instructive in the sense that it tells very little on the details of the underlying phenomena that lead to this relationship. In particular, it provides almost no information on the physical ingredients involved in the observed dynamo processes, e.g. whether they are driven by turbulence only ( $\alpha^{2}$ dynamo) or by differential rotation as well ( $\alpha \Omega$ or $\alpha^{2} \Omega$ dynamos), whether these processes actually take place in an interface layer like in the Sun or somewhere else in the convective zone, whether they trigger mainly axisymmetric low-order solar-like dynamo modes or more complex ones, whether the large-scale field is oscillatory or stationary, or whether the field has a feedback effect on the whole dynamics of the convective zone. Especially interesting is the case of fully convective stars; although theory predicts them to be much less active than non-fully convective ones from the fact that they lack an interface layer with a radiative interior and cannot trigger conventional dynamo processes (expected to concentrate in this layer), observations indicate that fully convective stars are just as active, suggesting that non conventional dynamos (such as distributed dynamos operating in the whole convective zone rather than being confined in the interface layer) could operate in both classes and make their activity levels and magnetic topologies very similar, irrespectively of their hosting an interface layer.

All this can however only be investigated with the help of the new indirect imaging techniques allowing the reconstruction of spatially resolved magnetic maps of stellar surfaces.

\section{Magnetic topologies versus rotation}

\subsection{Field topology}

Using indirect imaging techniques based on the principles of tomography and maximum entropy image reconstruction, one can succeed in reconstructing distributions of brightness and magnetic inhomogeneities in stellar photospheres. 
The performances and limitations of these techniques have been the subject of a large number of specific investigations in the last decade, for instance by Donati \& Brown (1997), Hussain et al. (2000) or Donati (2001) in the specific case of surface imaging of stellar magnetic topologies. These studies demonstrate in particular that, for cool active stars with moderate to fast rotation, sets of rotationally modulated Zeeman signatures in spectral line profiles obtained at high spectral resolution and collected throughout a stellar rotation cycle, can be used to recover, not only the location of magnetic regions at the surface of the star, but also, to a certain extent, the orientation of field lines within these regions. Automated software is used to translate the data sets into images of the stellar surface.

Such techniques have been applied a number of times already to reconstruct brightness distributions and magnetic topologies at the surface of rapidly rotating cool active stars (e.g. Donati \& Cameron 1997; Donati 1999; Donati et al. 1999; Donati et al. 2003a; Petit et al. 2003a, b). One of the most intriguing result that comes out of such studies is that most of the magnetic energy found at the surface of the star concentrates in regions in which the field is mostly azimuthal. Since regions of mostly azimuthal field can be safely distinguished, even from data sets including Zeeman signatures in circular polarisation only, from regions in which the field is either radial or meridional (e.g. Donati \& Brown 1997), one can conclude that this result is genuine and that such features must be produced by dynamo processes taking place in a subsurface shear layer, or possibly even distributed throughout the whole convective zone; one could indeed hardly imagine that toroidal field generated at the base of the convective zone as in the Sun could possibly show up as azimuthal field features at photospheric level.

Another striking feature of the magnetic topologies recovered to date for rapidly rotating cool active stars is that their azimuthal field regions tend to form rings of constant polarity, encircling the star at various latitudes. The most obvious example is that of the K1 subgiant of the RS CVn close binary system HR 1099, for which the azimuthal field component, very close to axisymmetry (see Fig. 1), features two rings of opposite polarities in the visible stellar hemisphere (a circumpolar ring of clockwise field and another one of counterclockwise field at a latitude of about $30^{\circ}$ ). Most other stars that have been magnetically imaged to date show, more or less clearly, similar azimuthal field surface patterns. The number of such rings seems to depend from one star to another, increasing apparently slightly with the average inverse Rossby number; in some stars, the axisymmetric field pattern is less obvious, hiding behind a non axisymmetric field component, but can be clearly evidenced by checking that the longitudinally averaged magnetic field polarity pattern with latitude repeats quite well from one epoch to the next (Donati \& Cameron 1997; Donati 1999; Donati et al. 2003a; Petit et al. 2003a, b).

This is interpreted as evidence that these intriguing azimuthal field structures witness the toroidal component of the large scale dynamo field. Long term monitoring of such magnetic images also suggest that these large scale dynamo fields may be oscillatory (Donati et al. 2003a); this characteristic, with the clear presence of axisymmetric field components (see above), argues strongly in favour that an $\alpha \Omega$ type dynamo is operating in very active stars. Again, detecting such toroidal field components at photospheric level implies that the underlying dy- 

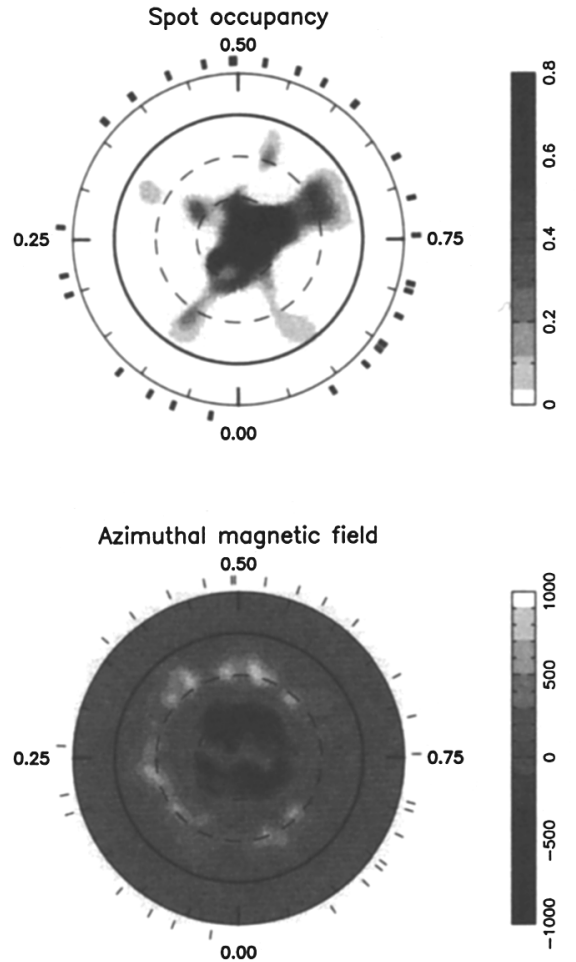
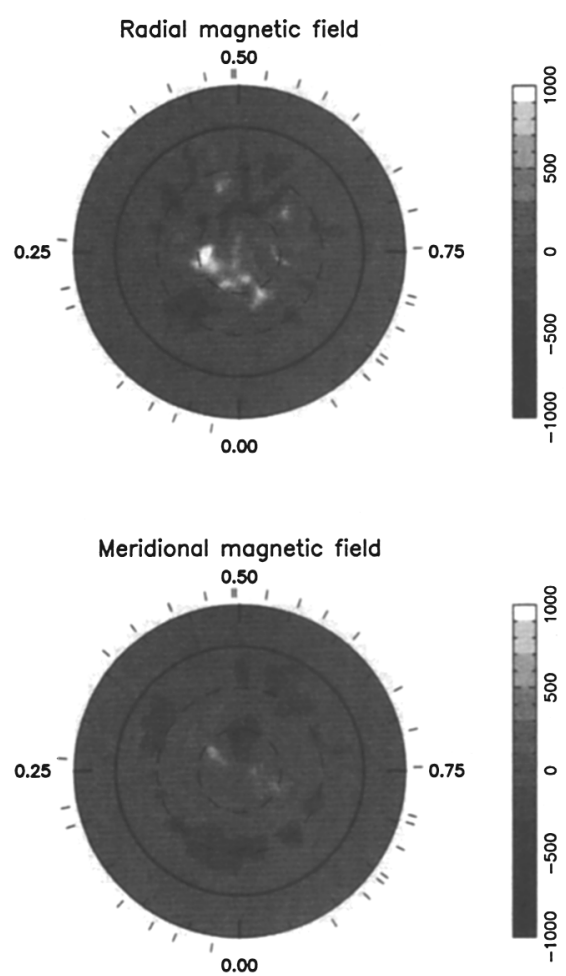

Figure 1. Maximum entropy brightness (upper left) and magnetic field images (in G) of the K1 subgiant of the RS CVn binary system HR 1099 at epoch 2001.99. These images are flattened polar projections extending down to a latitude of $-30^{\circ}$ (the bold and thin circles depicting the equator and the $30^{\circ}$ and $60^{\circ}$ latitude parallels respectively). The radial ticks around (and outside) each plot illustrate the phases at which the star was observed. Positive field values correspond to magnetic vectors directed outward, anticlockwise and poleward for radial, azimuthal and meridional field components respectively.

namo processes must be operating at the very least in a subphotospheric shear layer, and possibly even throughout the whole convective zone. The obvious conclusion is that such magnetic fields may indeed be sufficiently intense to produce a significant feedback effect on the global dynamics of the convective zone itself, at a much larger level than what is seen on the Sun, and could thus provide an appropriate explanation to the orbital period fluctuations observed in close binary systems. Such observations may potentially be extremely useful to test global dynamo models integrating self consistently all MHD aspects directly or indirectly linked to magnetic field generation.

Imaging methods other than standard maximum entropy were proposed and tested for reconstructing stellar surface magnetic field topologies for various kinds of stars. The possibility of using spherical harmonics expansions up to orders of several tens, with or without a priori assumptions on the local field structure (e.g. that the field is potential) proved to be particularly efficient at reconstructing structures that could not be reconstructed as images otherwise 
(e.g. Donati 2001; Hussain et al. 2001). They should be particularly interesting in the future for carrying out periodicity searches in the temporal evolution of magnetic topologies of stars other than the Sun, when long term series of spectropolarimetric data sets become more commonly available.

\subsection{Chromospheres and coronae}

By extrapolating the magnetic field derived from spectropolarimetric data from the photosphere of the star out to several stellar radii above the surface, one can obtain a rough idea of what the large scale coronal magnetic field looks like under simple approximations on the local field topology, e.g. that the field is potential (Jardine et al. 2002a; Hussain et al. 2002). Using different kinds of assumptions on the field distribution and orientation in the regions that cannot be imaged with the present data (e.g. the southern stellar hemisphere, the polar caps), one can even check the global magnetic topologies that result from these assumptions with other kinds of observational constraint (Jardine et al. 2002b), like for instance the coordinates of the massive prominences trapped in the largescale coronal loops of the star and detected as recurrent $\mathrm{H} \alpha$ transients (Cameron \& Robinson 1989a), or the X-ray light curves generated from the magnetically confined hot coronal plasma in corotation around the central star.

With such investigations, one could relatively simply explore what is the impact of rotation on the global structure of the extended magnetosphere, and in particular on the X-ray emitting corona. A possible study would be the one concerning the supersaturation of X-ray emission detected in ultrafast rotators. A possible interpretation of this effect is proposed by Jardine \& Unruh (1999); these authors suggest that, as the stellar rotation rate increases, centrifugal forces cause a rise in the pressure and density in the outer parts of the largest magnetic loops, breaking open previously closed field lines and destroying the associated X-ray emitting regions. Other totally different interpretations have also been proposed for this phenomenon (e.g. Stepien et al. 2001). By reconstructing surface magnetic topologies of stars with different rotation rates and $\mathrm{X}$-ray fluxes, and extrapolating these fields out to several stellar radii (as done by Jardine et al. 2002b), one could compare the predicted and observed X-ray emission and try to work out what exactly in the large-scale magnetic configuration triggers supersaturation.

\subsection{Surface differential rotation}

Additional constraints on dynamo processes can also be obtained by looking at the surface differential rotation that cool stars undergo. This can indeed be performed by investigating the short term variability that photospheric images of brightness and magnetic distributions are subject to on a time scale of a few days to a few weeks. Different methods have been proposed in the literature to perform such measurements (Donati \& Cameron 1997; Donati et al. 2000; Cameron et al. 2002; Petit et al. 2002; Donati et al. 2003b). Two of them can now be considered to have reached maturity; while the first one consists in merging all available spectra into a single set and finding out which photospheric shear and surface map produce the best fit to the data, the second one derives the recurrence rate of individual spots at the stellar surface from appropriate 
matched filter analyses of the spectral time series. Both methods are found to produce consistent and reliable results.

Summarising the main results obtained to date on single rapidly rotating young stars with a mass of about $1 \mathrm{M}_{\odot}$ (the post $\mathrm{T}$ Tauri star LQ Lup and the two Zero Age Main Sequence objects AB Dor and LQ Hya; Donati et al. 2000; Cameron \& Donati 2002; Donati et al. 2003b), one can derive that the photospheric shear evidenced in cool solar type stars is similar in sign (with polar regions rotating slower that equatorial regions) and strength (with a time of about 50 to $100 \mathrm{~d}$ for equatorial regions to lap the polar regions by one complete rotational cycle). In particular, this shear is found to be only weakly dependant on rotation (the studied stars rotating at a rate of about 20 to 80 times that of the Sun). The detected shear is found to be most of the time significantly larger when estimated from magnetic regions than from brightness regions (Donati et al. 2003b). Last but not least, repeating the experiment at different epochs yields that this photospheric shear seems to be variable with time (Cameron \& Donati 2002; Donati et al. 2003b), by amounts that can be as large as $200 \mathrm{mrad} \mathrm{d}^{-1}$.

\section{Magnetic feedback on rotation}

While rotation has an obvious consequence on the magnetic topologies and activity levels of cool stars, the opposite is also true, with magnetic fields having a significant feedback impact on stellar rotation. As first predicted by Schatzman (1962), a continuous outflow of gas from a magnetic star can produce a drain of angular momentum from the star far greater than that carried away if the gas were to feel no torque after leaving the stellar surface. Cool stars are thus expected to decrease their rotation rate considerably from the stage in their pre-main sequence history at which they dissipated their accretion disc up to some time after they reached the main sequence, in agreement with the very early observations of the rotational velocities in young open clusters suggesting what is now known as Skumanich's (1972) empirical law for magnetic braking of a star's rotation by a magnetic wind. This subject has been studied in detail in the past decades and was at the origin of numerous papers in the literature (e.g. Cameron \& Li 1994).

Centrifugal ejection of massive prominences, such as those repeatedly observed around young ultra fast rotators that just arrived on the main sequence (e.g. Cameron \& Robinson 1989a), can also be invoked to explain some of the magnetic braking observed for young low mass stars. Although these features are roughly similar in nature to their solar equivalent (sharing in particular the same temperature and density ranges), they turn out to be considerably more massive (by up to several orders of magnitude) than those of the Sun. A second difference is that they tend to condensate preferentially close to a place (the corotation radius) of unstable equilibrium, outside which centrifugal forces very easily expel them outwards. Observations of different stars (e.g. Cameron \& Robinson 1989b; Donati et al. 2000) demonstrate that the angular momentum loss from prominence ejections is sufficient to account for a significant fraction (if not most) of the magnetic braking observed at these stages of stellar evolution. 
In addition to braking down the whole rotation rate of cool stars on evolutionary timescales, magnetic fields can also be very efficient at modifying the internal rotational equilibrium of these stars on a much smaller time span. Magnetic fields are indeed suspected to be capable of redistributing angular momentum in the convective zones of cool stars as they progress along their activity cycle. The observation that surface differential rotation of a number of active stars is variable with time (e.g. Donati et al. 2003b) strongly argues in this favour. Note however that the small number of epochs at which differential rotation could be measured does not yet allow to conclude whether the temporal variations in the photospheric shear of these stars correlate well with other activity indexes. It therefore appears premature to conclude about a potential magnetic origin of the detected variability in the surface differential rotation of the few stars monitored to date. Nevertheless, magnetic activity remains by far the best candidate to explain such observations.

If one does assume that the time variability of stellar surface differential rotation is truly of magnetic origin, one can derive a number of potential implications on how angular momentum is distributed within the convective envelopes of cool stars. One can indeed reasonably speculate that total angular momentum within the convective envelope is globally conserved on time scales of at least a few decades. It implies in particular that any change in the rotation rate at the stellar equator must be related to a change of opposite sign in the rotation rate at the stellar pole, and that both changes relate are tied together through a relationship that depends both on the angular rotation law within the convective zone, and on the mass density radial profile of this envelope.

Using simple models for the angular rotation and internal structure within the convective envelope, straightforward analytical expressions (Donati et al. $2003 \mathrm{~b}$ ) indicate that the slope of $d \Omega$ versus $\Omega_{\text {eq }}$ relation (where $\Omega_{\text {eq }}$ and $d \Omega$ respectively denote the rotation rate at the equator and the difference in rotation rate between the equator and pole) should be about 5 if the angular rotation within the convective zone resembles that of the Sun (with angular velocity constant with radius) and ranging between 1.5 and 2 when the angular rotation within the convective zone is that expected for rapid rotators (with angular velocity constant along cylinders). From this conclusion, Donati et al. (2003b) derive that the variations in differential rotation detected in both young main sequence stars AB Dor and LQ Hya provide some observational evidence that the angular velocity field within their stellar convective zones is closer to that expected for rapid rotators than to that of the Sun.

In this context, it would imply that magnetic features, associated with the strongest shear, are anchored closer to the surface (i.e. at a depth where the difference between the equatorial and polar rotation rates $d \Omega$ is largest) than cool spots. This is at least in qualitative agreement with the conclusions of Donati et al. (2003a), claiming that magnetic features (and in particular those hosting predominantly azimuthal fields) must be formed very close to the surface in these stars. Further support about the idea that dynamo magnetic fields (and in particular their toroidal components) can be produced very close to the stellar surface comes from the recent discovery that the Sun features a subphotospheric layer with a strong radial gradient in angular rotation (Corbard \& Thompson 2002) that seems at least able to trigger dynamo action (Dikpati et al. 2002). 
The changes in kinetic energy associated to the differential rotation fluctuations can also be estimated with the same method. Assuming that this operation occurs over a typical timespan of a few years, one obtains that the power required to drive this change corresponds to about $1 \%$ of the total stellar luminosity in the case of AB Dor, and up to $10 \%$ in the case of LQ Hya. Although this sounds rather large already, one must keep in mind that only a small fraction of this amount is actually lost (i.e. radiated) in the process; it suggests that, even in the case of the star that exhibits the most drastic effect (i.e. LQ Hya), the corresponding power dissipated in the process does probably not exceed $1 \%$ of the stellar luminosity. A very rough estimate of the average magnetic field strength required within the convective zone to drive these changes yields values of about 10 to $20 \mathrm{kG}$ in the particular case of AB Dor and LQ Hya.

\section{Conclusion}

While magnetic fields of cool stars have long been known to be intimately connected with the rotation history of these objects, recent studies have brought new clues on some important details of the physical mechanisms involved in these processes. Magnetic images obtained from series of spectropolarimetric observations of Zeeman signatures from cool stars suggest for instance that dynamos of very active stars may also involve a component distributed throughout the whole convective zone, rather than a single one concentrating in the interface layer with the radiative interior as for the Sun.

Observations of massive prominences condensating at the corotation radius around rapidly rotating solar-type stars and trapped in the large-scale magnetic field suggest that magnetic braking of these stars in their late pre-main-sequence and early main-sequence stages is not only generated through a magnetic wind, but could also partly (if not mainly) be caused by the frequent expulsion of such circumstellar clouds. Magnetic images are also used to attempt modelling the large scale structure of the coronal field of cool stars, and to derive more insight on the detailed physics operating in their magnetospheres.

More recently, evidence was discovered that magnetic fields also have an impact on the global dynamics of the convective zones of cool active stars. Estimates of the differential rotation shearing the photospheres of these stars indicate that this shear varies with time, and could be linked to the magnetic activity cycle operating within the bulk of the convective zone. This discovery could explain the temporal fluctuations reported in the orbital period of close binary systems of active stars, as proposed by Applegate (1992).

All these research directions are very promising and should receive new impulse as next generation instruments dedicated to studying magnetic fields of stars of various types and brightnesses (such as ESPaDOnS, to be mounted at Canada-France-Hawaii Telescope at the end of year 2003) progressively appear on medium to large sized telescopes. This technical step forward should in particular help us study these effects in a much wider sample of stars, including targets of different ages, masses and rotation rates, than was possible up to now. 


\section{References}

Applegate J.H., 1992, ApJ 385, 621

Cameron A.C., Donati J.-F., 2002, MNRAS 329, L23

Cameron A.C., Donati J.-F., Semel M., 2002, MNRAS 330, 699

Cameron A.C., Li J., 1994, MNRAS 269, 1099

Cameron A.C., Robinson R.D., 1989a, MNRAS 236, 57

Cameron A.C., Robinson R.D., 1989b, MNRAS 238, 657

Corbard T., Thompson M.J., 2002, Solar Phys. 205, 211

Dikpati M., Corbard T., Thompson M.J., Gilman P.A., 2002, ApJ 575, L41

Dikpati M., Gilman P.A., 2001, ApJ 559, 428

Donati J.-F., 1999, MNRAS 302, 457

Donati J.-F., 2001, in: Boffin H.M.J., Steeghs D., Cuypers J. (eds.), "Astrotomography. Indirect Imaging Methods in Observational Astronomy". Lecture Notes in Physics 573. Springer, Berlin, p. 207

Donati J.-F., Brown S.F., 1997, A\&A 326, 1135

Donati J.-F., Cameron A.C., 1997, MNRAS 291, 1

Donati J.-F., Cameron A.C., Hussain G.A.J., Semel M., 1999, MNRAS 302, 437

Donati J.-F., Cameron A.C., Petit P., 2003b, MNRAS (submitted)

Donati J.-F., Cameron A.C., Semel M., et al., 2003a, MNRAS (submitted)

Donati J.-F., Mengel M., Carter B.D., et al., 2000, MNRAS 316, 699

Hussain G.A.J., Donati J.-F., Cameron A.C., Barnes J.R., 2000, MNRAS 318, 961

Hussain G.A.J., Jardine M.M., Cameron A.C., 2001, MNRAS 322, 681

Hussain G.A.J., van Ballegooijen A., Jardine M., Cameron A.C., 2002, ApJ 575, 1078

Jardine M.M., Unruh Y.C., 1999, A\&A 346, 883

Jardine M.M., Cameron A.C., Donati J.-F., 2002a, MNRAS 333, 339

Jardine M.M., Wood K., Cameron A.C., et al., 2002b, MNRAS 336, 1364

Mangeney A., Praderie F., 1984, A\&A 130, 143

Noyes R.W., Hartmann L.W., Baliunas S.L., et al., 1984, ApJ 279, 763

Petit P., Donati J.-F., Cameron A.C., 2002, MNRAS 334, 374

Petit P., Donati J.-F., Wade G.A., et al., 2003a, MNRAS (submitted)

Petit P., Donati J.-F., Wade G.A., et al., 2003b, MNRAS (submitted)

Prosser C.F., Randich S., Stauffer J.R., et al., 1996, AJ 112, 1570

Saar S.H., 1996, in: Strassmeier K.G., Linsky J.L. (eds.), IAU Symp. 176, "Stellar Surface Structure". Kluwer Academic Publishers, Dordrecht, p. 237

Schatzman E., 1962, A\&A 25, 18

Skumanich A., 1972, ApJ 171, 565

Stepien K., Schmitt J.H.M.M., Voges W., 2001, A\&A 370, 157

Strassmeier K.G., Fekel F.C., Bopp B.W., et al., 1990, ApJS 72, 191

Vaughan A.H., Preston G.W., 1980, PASP 92, 385 